\title{
A Three-Dimensional Evaluation Model of National Fragility Based on Dynamic Weighting
}

\author{
Qi-Fan Yang ${ }^{1}$, Xiao-Yan $\mathrm{Cao}^{1}$, Bing-Qian Liu ${ }^{1} \&$ Yuan-Biao Zhang. ${ }^{1,2,3}$ \\ ${ }^{1}$ Mathematical Modeling Innovative Practice Base, Jinan University, Zhuhai Campus, Zhuhai 519070, China \\ ${ }^{2}$ Packaging Engineering Institute, Jinan University, Zhuhai Campus, Zhuhai 519070, China \\ ${ }^{3}$ Key Laboratory of Product Packaging and Logistics of Guangdong Higher Education Institutes, Jinan \\ University, Zhuhai Campus, Zhuhai 519070, China
}

Correspondence: Yuan-Biao Zhang, Packaging Engineering Institute, Jinan University, Zhuhai Campus, Zhuhai 519070, China. E-mail: zybt@jnu.edu.cn

Received: March 13, 2018

doi:10.5539/jsd.v11n3p166

\author{
Accepted: April 27, $2018 \quad$ Online Published: May 30, 2018 \\ URL: https://doi.org/10.5539/jsd.v11n3p166
}

\begin{abstract}
Nowadays, climate change has become an increasingly important factor that influences the national development. In this paper, we propose the three-dimensional model based on dynamic weighting to measure national fragility, while taking into account a series of climatic factors like temperature, rainfall et al. Our model includes 20 indicators which can be divided into economic factors, social factors and environmental factors. We first divided all indicators into cost-type, benefit-type and moderate indicators, and normalized them based on different types of indicators. Then, combining modified entropy weight method and AHP, the weights of 20 indicators and three factors in the evaluation model are defined. In the three-dimensional evaluation model, we use the length of the evaluation curve to evaluate the national fragility and measure the balance of the three factors with the angle between the curve and the diagonal of the model. Moreover, since countries at different stages of development have different development focuses, we have developed an "S-type" function to dynamically measure the different emphasis on the degree of national fragility and the balance of the three evaluation factors. Then, we calculate the comprehensive fragility index by giving different weights for the degree of national fragility and the balance of the three factors. Finally, we use two different countries which are China and Sudan to verify the rationality of the model. The results show that our model can reasonably measure the fragility of countries in different development levels, which also proves its adaptability and practicability.
\end{abstract}

Keywords: three-dimensional model, dynamic weighting, national fragility, climate change

\section{Introduction}

A country or state is fragile when it doesn't have enough capacity to supply the fundamental essentials to its individuals. It is essential for every country to assess the national fragility which is used to not only develop better national development plans but also accelerate the country's economic development. Many organizations evaluate it mainly through a country's social and economic indicators as well as government capabilities. For example, the FFP organization measures the fragile degree of a country through 12 indicators, that is, Security Apparatus, Factionalized Elites, and Group Grievance et al. (CIFP, 2006).

Carleton University defines the CIFP index to measure the national fragility from four dimensions of economy, society, population and environment (Huang, 2016). Furthermore, not only CIFP but also other Index systems such as FFP, CRISE and CSP measure the fragile degree of a country do not take into account the impact of climate change on national fragility (Carment, Prest \& Samy, 2009; Stewart \& Brown, 2009; Marshall \& Cole, 2014). What's more, some researchers made a comparative summary of the multiple index systems in the Western academic community on measuring the country's fragility and concluded that there are several problems in each link which will further exacerbate the country's fragility (Liu \& Wu, 2016; Zhu \& Hao, 2017 ). Sanín studied seven kinds of assessment indexes that measure national fragility and criticized the national performance index because almost no information about performance ranking was collected (Sanín, 2011).

Nowadays, the climate change has become a hot concern point. Climate change interacts with the fragility of a state. Deteriorating climate change, like decreasing rainfall and increasing temperature, has great influence on 
human lives through economic, social and environmental effects, which will increase the fragile degree of a country. Besides, if a country is fragile, its population will be more vulnerable to cope with the climate change; even sometimes the change is small. That means it is a vicious circle system. What's more, as the climate change is getting serious, it is vital to measure a country's fragility and related climatic impact.

For this reason, we propose a 3D model based on the dynamic weighting for establishing an evaluation system that incorporates environmental indicators to assess national fragility. We assume that the selected regions will not occur major natural disaster in the future and the trends in selected regions are determined by various indicators. These assumptions ensure that there will be no sudden change in the indicators of the selected area and that the indicators of the area will determine the development trend in the area. Then our model selects about 20 indicators in all aspects to assess national fragility.

Our model's purpose is to include the impact of climate change on the country's fragility and make it more scientific and comprehensive. What's more, it also can also help countries at different stages of development formulate development strategies that are tailored to their national conditions according to their national fragilities.

\section{Method}

\subsection{Determine Indicator System}

The evaluation criteria for fragility are different in different studies. The World Bank has developed two fragility indices, the Resource Allocation Index and the Global Governance Index (The World Bank, 2005). Based on the above analysis, we select 20 indicators, propose the Economic Development Factor (EDF), Social Stability Factor (SSF) and Environmental Sustainability Factor (ESF), and establish a three-dimensional index system to reflect the fragility of a country. The following Figure 1 shows our indicator system.

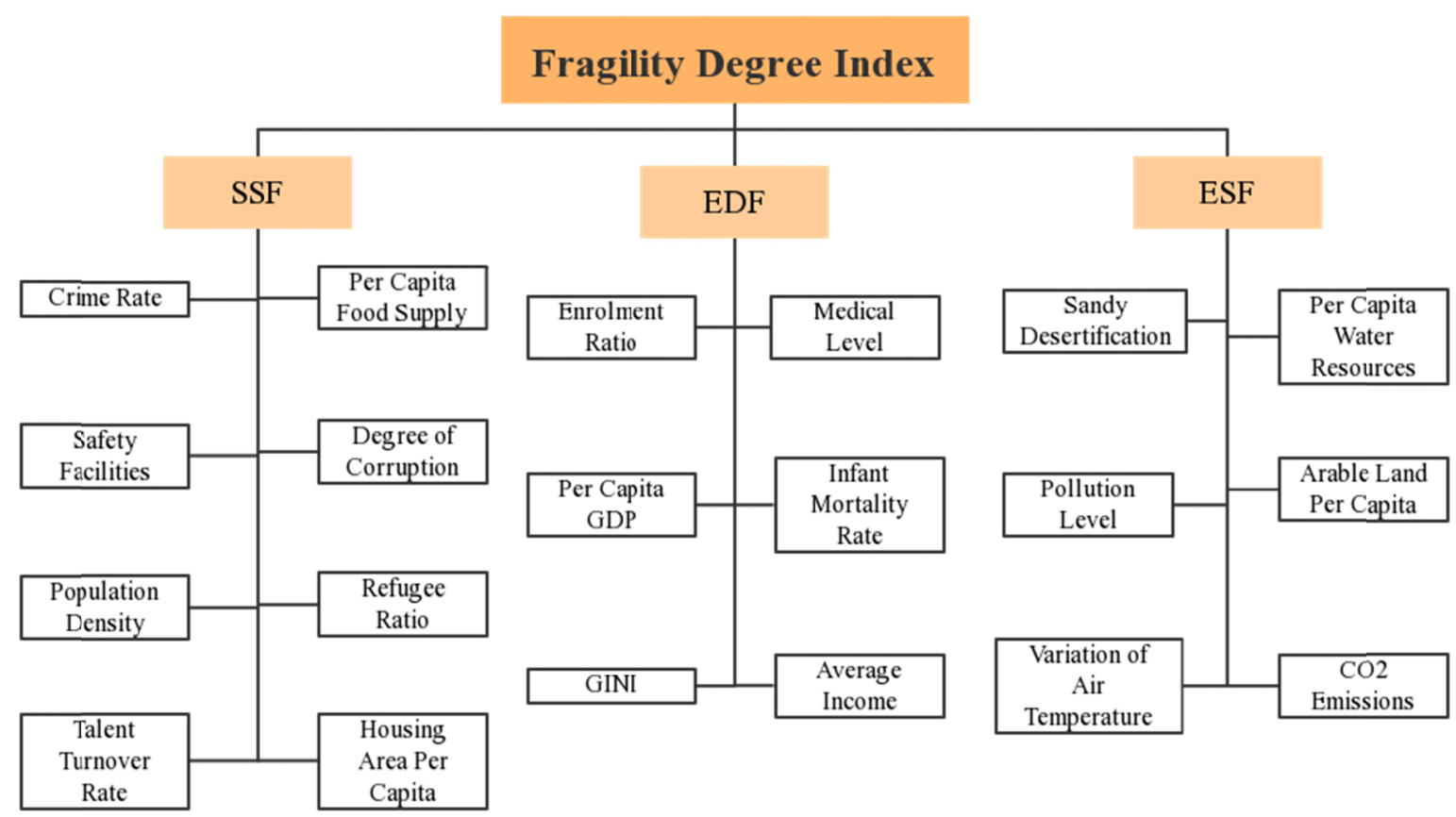

Figure 1. The three-level indicator system

From our indicator system, we measure these indicators in different ways. Here we explain some of these indicators.

Firstly, we adopt the "Corruption Perceptions Index" to measure the degree of Government Corruption. Secondly, we adopt the "Crime Index" to measure the crime rate of a state, which is public data from the global city database "cumbeo" website. And then, we adopt the annual National Security expenditure to measure Security Apparatus of a country. Finally, we adopt the "Pollution Index" to measure the pollution level of a state, which is public data from the global city database "cumbeo" website. 


\subsection{Data Normalization}

As the dimension of all indicators is different, these indicators cannot be compared directly. We converted all the data to number between 0 and 1 .

All indicators can be divided into three classes, cost-class indicators, benefit-class indicators and moderate indicators. The cost-class indicators include crime rate, degree of corruption, talent turnover rate, sandy desertification, infant mortality rate, GINI, pollution level and refugee ratio. The benefit-class indicators include safety facilities, housing area per capita, per capita food supply, medical level, average income, enrolment ratio and per capita GDP. The moderate indicators include population density, variation of air, temperature and $\mathrm{CO} 2$ emissions, arable land per capita and per capita water resources.

As a result, we use three different approaches to normalize the three types of data.

The $j-t h$ index of the $i-t h$ is represented by $x_{i j}$.

$x_{i j} \in$ benefit-class index

$$
r_{i j}= \begin{cases}0, & x_{i j} \in\left[0, a_{i}\right] \\ 1-K_{i} \times e^{x_{i j}-a_{i}}, & x_{i j}>a_{i}\end{cases}
$$

Where, $K_{i}$ is a specific parameter, $K_{i}>0$.

$x_{i j} \in$ cost-class index

$$
r_{i j}=\left\{\begin{array}{l}
1, \quad x_{i j} \in\left[0, b_{i}\right] \\
1-\frac{S_{i}\left(x_{i j}-b_{i}\right)^{2}}{1+S_{i}\left(x_{i j}-b_{i}\right)^{2}}, x_{i j}>b_{i}
\end{array}\right.
$$

Where, $S_{i}$ is a specific parameter, $S_{i}>0$.

$x_{i j} \in$ moderate index

$$
\mathrm{r}_{i j}=\frac{k e^{-\frac{x_{i j}}{2}} x_{i j}^{\frac{n}{2}-1}}{2^{\frac{n}{2}} \int_{0}^{+\infty} t^{x_{i j}-1} e^{-t} d x}
$$

\subsection{Determine Weight Models}

\subsubsection{Improved Entropy Weight Model}

We first use the entropy method to determine the weight of 20 third-level indicators. Entropy method is an excellent way to determine the weight of the criterion.

We can calculate the entropy value $e_{j}$.

$$
e_{j}=-k \sum_{i=1}^{m} p_{i j} \ln p_{i j}
$$

Where, $k=\frac{1}{\ln m}$, the $p_{i j}$ is $j-t h$ the index of the $i-t h$ sample. 


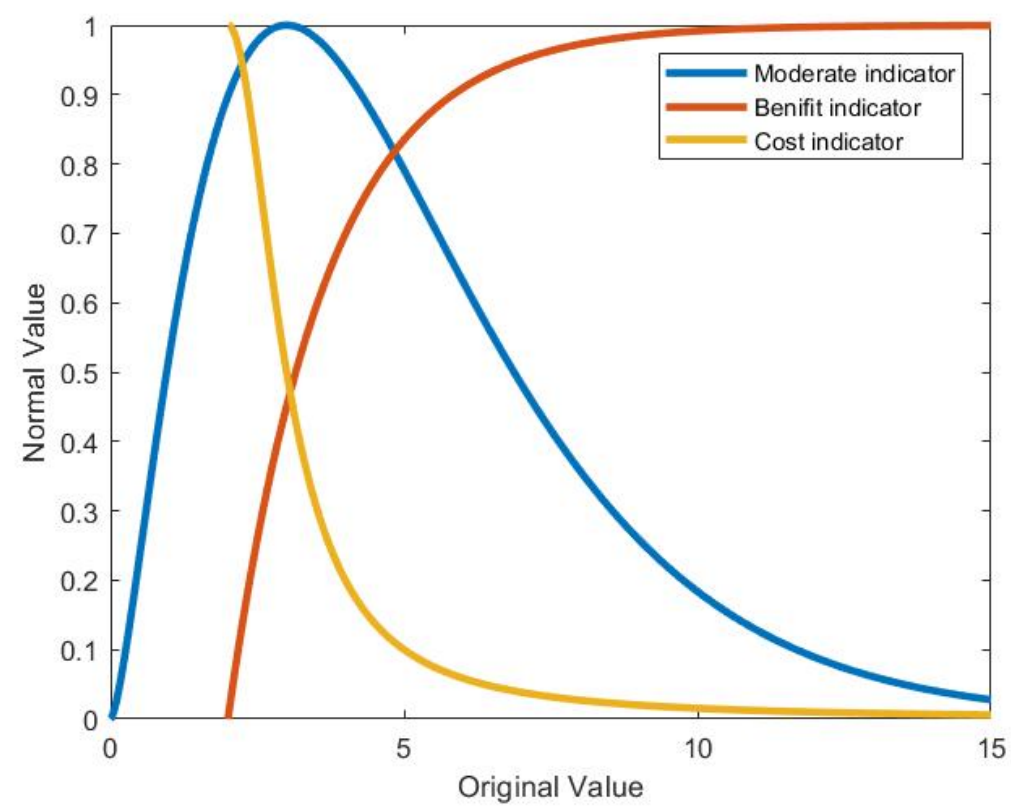

Figure 2. The curves of normalized functions

Then the weight $\mu_{j}$ is reflected by

$$
\mu_{j}=\frac{1-e_{j}}{\sum_{j=1}^{n}\left(1-e_{j}\right)}
$$

Calculated by MATLAB, we found that some indicators are highly significant, but their weights tend to be 0 as their smaller dispersion. Thus we modify entropy weight model combining grey relational analysis $(\mathrm{Li} \& \mathrm{Fu}$, 2007), that is,

The components $r_{0 i}$ of reference sequence $r_{0}$ is represented by

$$
r_{0 i}=\max _{1 \leq i \leq m}\left\{r_{i j}\right\}, i=1,2, \mathrm{~L}, n
$$

Let $\zeta_{j}(k)$ denote the gray relational coefficient, which is calculated by

$$
\zeta_{j}(k)=\frac{\min _{1 \leq j \leq m} \min _{1 \leq k \leq n}\left|r_{0 k}-r_{j k}\right|+\beta \max _{1 \leq j \leq m} \max _{1 \leq k \leq n}\left|r_{0 k}-r_{j k}\right|}{\left|r_{0 k}-r_{j k}\right|+\beta \max _{1 \leq j \leq m} \max _{1 \leq k \leq n}\left|r_{0 k}-r_{j k}\right|}
$$

Where $\mathrm{j}$ reflects the $\mathrm{jth}$ indicator, $\mathrm{k}$ reflects the $\mathrm{kth}$ optimal indicator, $\beta$ reflects the resolution ratio, $0<\beta<1$, here, we determine $\beta=0.5$.

The combined weight of each indicator is calculated by

$$
p_{0 j}=\sum_{k=1}^{n} \mu_{j} \zeta_{j}(k)
$$

Where, $\mu_{j}$ is the entropy weight, $p_{0 j}$ reflects the combined weight.

2.3.2 Weight Model Based on AHP

We utilize AHP to determine the weights of Economic Development Factor (EDF), Social Stability Factor (SSF) and Environmental Sustainability Factor (ESF). 
Analytic Hierarchy Process (AHP) is a simple approach to determine the weight of different levels, especially for the quantitative problem analysis (Saaty, 2008). The table 1 below shows the defined weights,

Table 1.Weights of three factors

\begin{tabular}{cccc}
\hline FDI & EDF & SSF & ESF \\
\hline SSF & 1 & 1 & 2 \\
EDF & 1 & 1 & 2 \\
ESF & $1 / 2$ & $1 / 2$ & 1 \\
\hline
\end{tabular}

Calculated by MATLAB, $\mathrm{CR}=\mathrm{CI} / \mathrm{RI}=0.0158<0.1$. So the coherence of the matrix is qualified.

\subsection{Three-Dimensional Fragility Metric}

As is stated before, we construct a Three-Dimensional Fragility Metric (TFM) to evaluate the fragility of a country, which is incorporated with EDF, SSF and ESF.

Three-Dimensional Fragility Metric is measured by Fragility Degree Index (FDI) and Stability Equity Index (SEI). FDI indicates the degree of fragility reduction in a country, and SEI indicates a balanced level of economic, social and environmental stability

In order to determine the metric of FDI and SEI, we follow several steps,

Step 1

We first define the actual index vector $\stackrel{\text { umu }}{O}$ and ideal index vector $\stackrel{\text { umu }}{O}$.

$$
\begin{gathered}
\stackrel{\text { unu }}{O C}=(E D F, S S F, E S F) \\
\stackrel{\text { unu }}{O G}=\left(E D F_{\text {ideal }}, S S F_{\text {ideal }}, E S F_{\text {ideal }}\right)
\end{gathered}
$$

Step 2

Then, as these three factors have the different contribution to the value of TFM, we redefine the weight vector $\left|O G_{\text {weight }}\right|$ and $\left|O C_{\text {weight }}\right|$, their length can be represented by

$$
\begin{gathered}
\left|\mathrm{uUr}_{\text {weight }}\right|=\sqrt{\left(\alpha_{1} E D F_{\text {ideal }}\right)^{2}+\left(\alpha_{2} S S F_{\text {ideal }}\right)^{2}+\left(\alpha_{3} E S F_{\text {ideal }}\right)^{2}} \\
\left|\mathrm{uur}_{\text {weight }}\right|=\sqrt{\left(\alpha_{1} E D F\right)^{2}+\left(\alpha_{2} S S F\right)^{2}+\left(\alpha_{3} E S F\right)^{2}}
\end{gathered}
$$

In the above equation, we follow several steps to define $\alpha_{i}$

We first define the weight mapping based on the model,

$$
f: w_{i} \rightarrow \alpha_{i}, i=1,2,3
$$

Where, $w_{i}$ reflects the weight that is calculated by AHP.

Then considering that different countries have different emphasis on economy, society and environment, we define the weight coefficient between different factors $m_{i}$, it is calculated by

$$
m_{i}=\frac{w_{i}}{w_{j}}, i=1,2 ; j=3
$$

In the three-dimensional model, the relationship between $m_{i}$ and $\alpha_{i}$ can be represented by 


$$
\left\{\begin{array}{l}
\sum_{j=1}^{n}\left(\alpha_{i}\right)^{2}=1 \\
\frac{\alpha_{i}}{\alpha_{j}}=m, i=1,2 ; j=3
\end{array}\right.
$$

Therefore, $\alpha_{1}, \alpha_{2}, \alpha_{3}$ can be calculated by

$$
\left\{\begin{array}{l}
\alpha_{1}=\frac{m_{1}}{\sqrt{m_{1}^{2}+m_{2}^{2}+1}} \\
\alpha_{2}=\frac{m_{2}}{\sqrt{m_{1}^{2}+m_{2}^{2}+1}} \\
\alpha_{3}=\frac{1}{\sqrt{m_{1}^{2}+m_{2}^{2}+1}}
\end{array}\right.
$$

Step 3

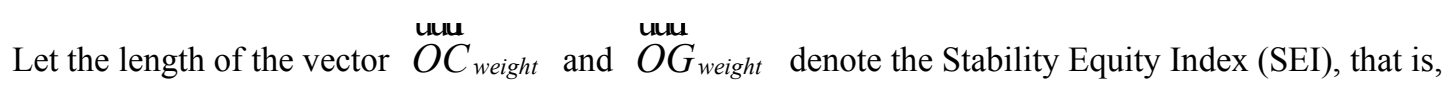

$$
F D I=\left|O C_{\text {weight }}\right|
$$

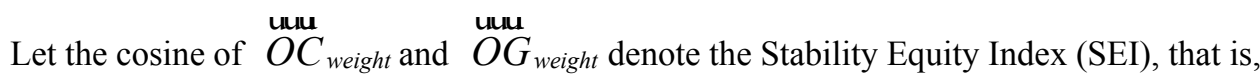

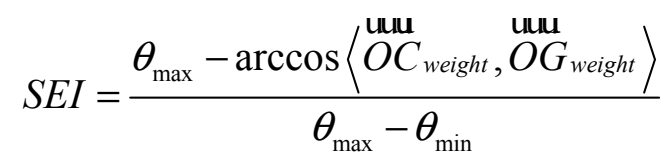

Where, $\theta$ is deviation angle from balanced stability condition.

A higher FDI represents a lower reduction of fragility, which means the country is becoming less fragile. A higher SEI reflects an equal development of stability.

To comprehensively measure the degree of national fragility and the balance of various indicators, we define the Comprehensive Fragility Index (CFI) to measure the fragility of the country, combining Fragility Degree Index (FDI) and Stability Equity Index (SEI). The CFI is calculated by

$$
C F I=(1-\beta) S E I+\beta F D I
$$

Where the determination of $\beta$ is a critical issue.

As the country's economic development, the state should be the more balanced development of various indicators. Therefore, to make our model more widely available, we propose a three-dimensional evaluation model based on dynamic weighting.

As is stated before, we propose "S" curve as a variable weight function.

$$
\begin{cases}0, & x<a \\ \frac{1}{2}-\frac{1}{2} \sin \frac{\pi}{b-a}\left(x-\frac{a+b}{2}\right), & a \leq x \leq b \\ 1, & x>b\end{cases}
$$

Where $\mathrm{a}, \mathrm{b}$ are specific parameters. In this paper, $\mathrm{a}=0, \mathrm{~b}=1 . \mathrm{X}$ represents the value of EDF, and $\mathrm{y}$ is the dynamic weight $\beta$. 
Calculated by MATLAB, the weight of different countries is defined.

\subsection{Experiment}

We choose the 5th most fragile state-Sudan as our research object. Located in the northeast of Africa, the eastern part of the Sahara, Sudan is one of the least developed countries declared by the United Nations. Drought is the fundamental characteristics of Sudan's climate, the capital Khartoum has the name of "world's stove", with the average annual temperature above $30^{\circ} \mathrm{C}$. To verify the rationality of our model and also to show that the model is suitable for different development states, we also study the 85th most vulnerable country in China. Compared with Sudan, the situation in China is much better.

For measuring the different countries' fragility, the experimental flow is as follows:

(1) Data preprocessing

According to the characteristics of various indicators, we use three different approaches to normalize the three types of data and convert all the data to number between 0 and 1 for next step.

(2) Calculating the coordinates of the 3D model

We modify entropy weight model combining grey relational analysis for determining the weight of different indicators. Then, we can get the Economic Development Factor (EDF), Social Stability Factor (SSF) and Environmental Sustainability Factor (ESF) of the different country as the coordinates of the 3D system.

(3) Defining the three-dimensional model

In this section, we first calculate FDI, SEI and SSF of Sudan and China based on collected data. The following table 2 shows reckoning in each flow.

Table 2.The index values of the two countries

\begin{tabular}{ccccccc}
\hline Country & CFI & FDI & SEI & ESF & SSF & EDF \\
\hline Sudan & 0.392 & 0.318 & 0.884 & 0.298 & 0.339 & 0.310 \\
China & 0.596 & 0.552 & 0.653 & 0.648 & 0.478 & 0.388 \\
\hline
\end{tabular}

Then, according to different economic development levels of two countries, $\beta$, the weight of FDI is defined. For Sudan, it is 0.112 . For China, $\beta$ is set to 0.421 . Therefore, we can obtain the comprehensive fragile degree (CFI) of Sudan and China, which are 0.3923 and 0.5961 respectively.

After that, we can establish the three-dimensional model to measure the fragility of China and Sudan more specifically and precisely. In this model, the EDF, SSF and ESF of each country are taken as three axes.Then, we define the weight mapping based on the model. The 3D models of Sudan and China are as follows: 


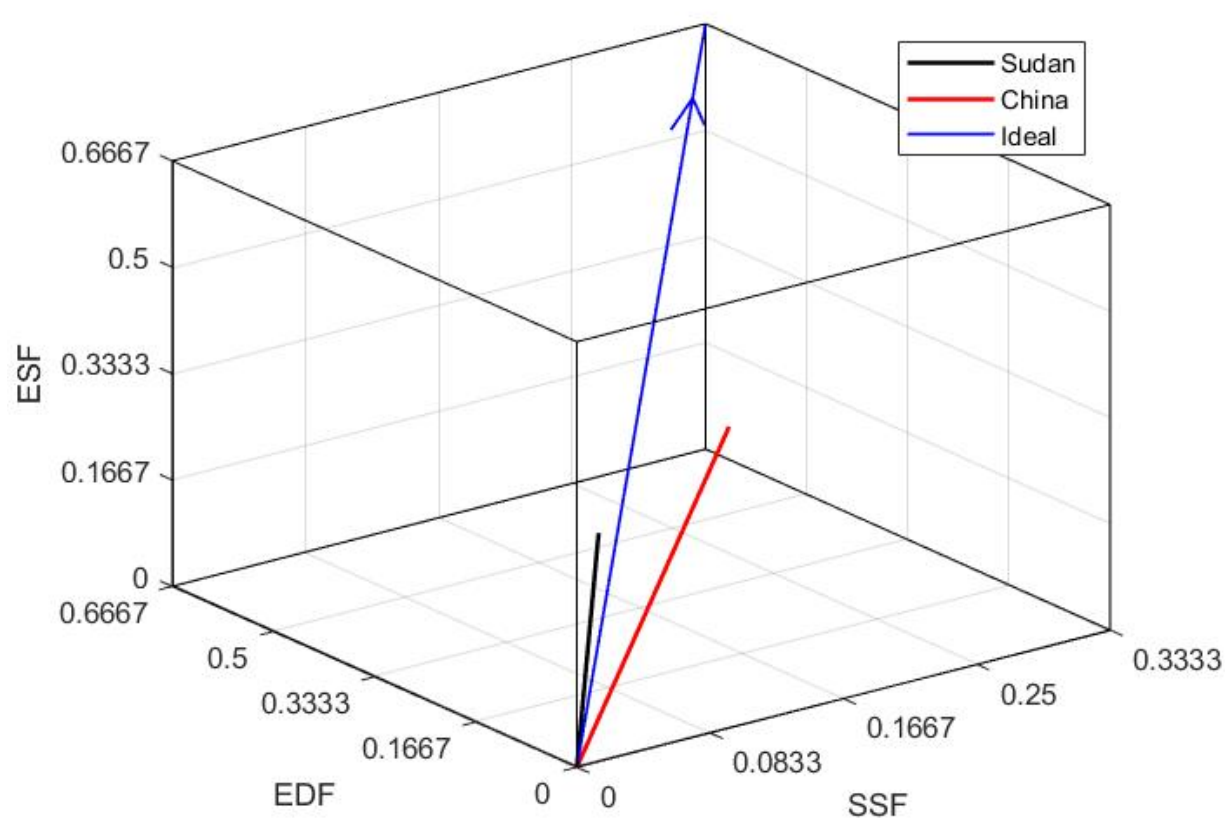

Figure 3. The three-dimensional evaluation models of Sudan and China

In the three-dimensional model, let the length of vector denote the Fragility Degree Index (FDI), which calculated by equation 17. Let the cosine indicate the Stability Equity Index (SEI), which calculated by equation 18. In Figure 3, the blue line with the arrow represents the ideal optimal FDI value, the black line reflects the current FDI of Sudan, and the red line is the current FDI of China. The $\mathrm{x}, \mathrm{y}, \mathrm{z}$ axis represent EDF, SSE, and ESF respectively. From the Figure 3, we can analyze the fragility of two countries clearly and visually.

\section{Results and Discussion}

In our evaluation model, Sudan is more fragile than China. Further analyzing, we can find that although the FEI of Sudan is lower than that of China, Sudan has a higher SEI which is 0.884 . That means, Sudan is more balanced than China in the development of environment, society and economy. The values of EDF, SSF and ESF in Sudan is almost the same, with $0.298,0.339$ and 0.310 respectively.Among three values of EDF, SSF and ESF, the EDF is the lowest in Sudan, which demonstrates that the economic development level in Sudan is pretty slow. The economy is a vital factor that influences the fragility of Sudan.

As for China, the condition is a little different. Although China is more stable than Sudan, its SEI value is much lower. In other words, comparing with Sudan, China is less unbalanced in the development of environment, society and economy. There is a significant difference between EDF, SSF and ESF. The EDF is the highest with 0.648 , which shows that the governments in China pay more attention to developing economy while ignoring the effects of SSF and ESF.

Further analyzing, we found that the different fragile condition has a lot to do with the local conditions.

(1) Due to the influence of geographical, environment and war, Sudan's three indicators are deficient, which means Sudan's society, economy and environment need to thrive. This situation will require the Sudanese government to raise social stability, reduce the number of refugees, increase investment in safety facilities, vigorously develop the economy and improve the local environment.

(2) Compared to Sudan, China has much higher social and economic indicators than Sudan's but with lower environmental indicators. Therefore, in the three-dimensional cube model, China's SEI indicator is at a low level. The calculation of CFI should take more into account the factors of balanced development. From the table, we can see that the environment in China cannot be synchronized with social and economic development. The imbalances in this development have exacerbated China's fragility. Therefore, the Chinese government should strengthen its environmental governance and protection while carrying out its economic development.

(3) For underdeveloped countries like Sudan, their social and economic development is still sluggish. Their 
governments should pay more attention to maintaining national stability and promoting social development. Therefore, our model is based on a dynamic weighting function to give more weight to the FDI that represents the development of the country when calculating Sudan's fragility. Compared with the Sudan, China's security has become more stable, and its economic development has been more rapid. However, we can see from the 3D model that China's environmental indicators are significantly lower than the other two indicators. Under such circumstances, the imbalance in the development status of various indicators is a key factor leading to the country's vulnerability. Therefore, according to the dynamic weighting function, we give more weight to the SEI that represents a balanced development for countries with better development status and promotes the government to pay more attention to balanced development.

This paper improves the entropy weight method based on the grey relational degree and objectively analyzes the importance of the three indicators of fragility, the Economic Development Factor (EDF), the Social Stability Factor (SSF) and the Environmental Sustainability Factor (ESF). This way will provide a scientific basis for formulating development plans. Nowadays, the deterioration of the global climate is getting more and more intensified, and its influence on the development of the country is gradually increasing. Therefore, this paper innovatively adds several climate indicators, such as temperature and precipitation, so that our index system can more truly and reasonably reflect the current state of development of the country. At present, the existing literature on the calculation of the country's fragility index system mostly accumulates the scoring method but does not score the fragility by way of comprehensive weight. In view of this, this paper divides the fragility into three dimensions: economic development factors, social stability factors, and environmental sustainability factors, and selects 20 indicators to measure the country's fragility comprehensively. Moreover, in data analysis, compared with the single normalization method of other papers, we divide the indicators into extremely large, very small, and intermediate types, and introduce three types of normalized functions. Then we adopt the three types of normalized functions to avoid the subjectivity that is caused by artificial empowerment. The entropy weight method is used to calculate the weight, and at the same time, considering that the entropy weight method has limitations on the calculation of the weight of the smaller fluctuation index, the gray correlation is introduced to improve, and the weight of the 20 indicators on the three major factors is obtained. Then, combine AHP with comprehensive weights.

Then we propose a three-dimensional model based on dynamic weights. In recent years, although Western scholars have studied a variety of evaluation indexes to assess the country's fragility, most of them only measured the degree of fragility which is the current state, and they do not give clear guidance on the future direction of a country. From this, this paper innovatively proposes a three-dimensional evaluation model, which classifies the country's fragility into three categories: economic development factors, social stability factors, and environmental sustainability factors. Using the space vector angle, we can obtain a deviation from the current development direction and ideal development direction of a country. We represent the degree of deviation for the development of equilibrium in this paper. Besides, the current development level of the country is measured by the vector module length. Then, we consider that a country has different emphasis on development balance and development level at the different stages of development. Therefore, we introduce an "S" dynamic weighting function to weight these two indicators. Using the national data of the Sudan and China for model checking, the findings are in line with reality and can point out the current development defects of the two countries here, namely the causes of vulnerability, and can give clear directions for their future development. It is worth mentioning that this model can also provide a reference for the evaluation of other dynamic developments.

\section{Conclusion}

For measuring the impact of environmental factors such as climate on the national fragility, this paper establishes a three-dimensional model based on dynamic weighting to evaluate the national fragility. This model uses 20 indicators for establishing a three-dimensional social, economic and environmental evaluation system to evaluate the national fragility. In this 3D model, the scale is used to indicate the degree of national development and the angle indicates the balance of national development. After that, it shows the degree of social fragility by the integrated value of two indicators. At the same time, the dynamic weighting function enables developed countries to pay more attention to the comprehensive development of all the indicators according to different development priorities of different countries, which makes the model more scientific. From our experiment, the calculation examples show that the three-dimensional model based on dynamic weighting is efficient, reliable and also has reasonable practicability and generalization.

\section{References}

Carment, D., Prest, S., \& Samy, Y. (2009). Security, Development and the Fragile State. Security, development, 
and the fragile state: bridging the gap between theory and policy. Routledge.

CIFP. (2006). Failed and Fragile States. Retrieved February 10, 2018, from https://carleton.ca/cifp/?p=34

Huang, Y. B. (2016). An analysis of the rule-of-law thinking in fighting against corruption - from the perspective of"set exact off"and corruption perceptions index. Journal of Beijing Police College, 1, 20-29. https://doi.org/10.16478/j.cnki.jbjpc.20160114.006

Li, G. L., \& Fu, Q. (2007). Grey Relational Analysis Model Based on Weighted Entropy and its Application. International Conference on Wireless Communications, NETWORKING and Mobile Computing (Vol.17, pp.5500-5503). IEEE. https://doi.org/10.1109/wicom.2007.1347

Liu, T. X., \& Wu. T. (2016). Issues of Assessment Criteria for Fragile Countries. The Science of Leadership Forum, (13), 17-26. https://doi.org/10.19299/j.cnki.42-1837/c.2016.13.002

Marshall, M. G., \& Cole, B. R. (2008). Global report on conflict, governance and state fragility 2008. Foreign Policy Bulletin, 18(1), 3-21. https://doi.org/10.1017/s1052703608000014

Saaty, T. L. (2002). Decision making with the analytic hierarchy process. International Journal of Services Sciences, 1(1), 83-98. https://doi.org/10.1504/ijssci.2008.017590

Sanín, F. G. (2011). Evaluating state performance: a critical view of state failure and fragility indexes. European Journal of Development Research, 23(1), 20-42. https://doi.org/10.1057/ejdr.2010.53

Stewart, F., \& Brown, G. (2009). Fragile states. University of Oxford. Centre for research on inequality, human security and ethnicity (CRISE).

The World Bank. Fragile states: Good Practice in Country Assistance Strategies Operation and Country Services, December 19, 2005, No.34790.

Zhu, J., \& Hao, Q. Y. (2017). "Fragile State" and Civilized Mission: Reassessment of National Fragility Index. Probe (05), 157-164. https://doi.org/10.16501/j.cnki.50-1019/d.2017.05.025

\section{Copyrights}

Copyright for this article is retained by the author(s), with first publication rights granted to the journal.

This is an open-access article distributed under the terms and conditions of the Creative Commons Attribution license (http://creativecommons.org/licenses/by/4.0/). 